\title{
RECURSIVE PROCESSING OF Nested StRUCTURES IN MONKEYS? TWO ALTERNATIVE ACCOUNTS
}

\author{
E-LETTER \\ Yair Lakretz \\ Cognitive Neuroımagıng Unit \\ CEA, INSERM, Université Paris-Sud, Université Paris-Saclay \\ NeuroSpin center \\ 91191 Gif-sur-Yvette, France \\ yair.lakretz@gmail.com \\ Stanislas Dehaene \\ Cognitive Neuroimaging Unit \\ CEA, INSERM, Université Paris-Sud, Université Paris-Saclay \\ NeuroSpin center \\ 91191 Gif-sur-Yvette, France \\ Collège de France \\ 11 Place Marcelin Berthelot, 75005 \\ Paris, France
}

Ferrigno et al. [2020] introduced an ingenious task to investigate recursion in human and non-human primates. Participants view a touch screen with four bracket signs, such as \} \{ ] and [, presented at random positions. During training, they are rewarded for touching these items in a center-embedded sequence, either $\{()\}$ or $\{[]\}$. At test time, generalization to new sequences is examined: given a new display of ( ) [ ], do the participants spontaneously produce a center-embedded structure such ( [ ] ) or [ ( ) ] ? American adults, Tsimane adults, and 3-5 year-old children did. Macaque monkeys required additional training, but two out of three eventually showed good generalization and scored above many Tsimane and child participants. Moreover, when tested on sequences composed of new bracket signs such as $<$ and $>$, the monkeys still showed good performance. The authors thus concluded that recursive nesting is not unique to humans.

Here, we dispute the claim by showing that at least two alternative interpretations remain tenable. We first examine this conclusion in light of recent findings from modern artificial recurrent neural networks (RNNs), regarding how these networks encode sequences. We show that although RNNs, like monkeys, succeed on demanding generalization tasks as in Ferrigno et al., the underlying neural mechanisms are not recursive. Moreover, we show that when the networks are tested on sequences with deeper center-embedded structures compared to training, the networks fail to generalize. We then discuss an additional interpretation of the results in light of a simple model of sequence memory [Botvinick] and Watanabe, 2007|.

RNN-based Language Models are trained to predict the next word in a large corpus of natural- or artificial-language data, such as the 'bracket' language (aka, Dyck languages; Suzgun et al. 2019). At each time step, the model outputs a probability distribution for the next item in the sequence given all preceding words, and can therefore be evaluated on tasks such as Ferrigno et al. 's. In natural language, constructions with center embeddings are mostly bound to one level of nesting [Karlsson, 2007], in which an inner grammatical dependency is embedded within a longer one (e.g. 'The dog the cats chase runs away'). RNNs trained on a large natural-language corpus can process such constructions [Linzen et al. 2016]. However, we recently showed that they do not rely on genuine recursion, but use different neural mechanisms for each of the two structural dependencies [Lakretz et al., 2019, 2021a]. The long-range dependency is processed by a specialized neural circuit composed of: (1) a 'syntax' unit, whose activity tracks the structure of the long-range dependency, thus binding the corresponding subject and verb; and (2) one or two grammatical-feature units, which carry grammatical information such as number or gender across the long delay between the subject and 
the corresponding verb, and whose activity is controlled by the syntax unit. In contrast, the short-range agreement is processed by a separate set of units without any strong delay activity. Importantly, these short- and long-range mechanisms operate independently and therefore do not constitute a recursive process. Moreover, the emerging long-range mechanism comprises only a very small number of units. This was shown to limit the capacity of the network to a single long-range dependency [Lakretz et al., 2020, 2021a].

In a recent study [Lakretz et al., 2021b], we trained RNNs on artificial data sampled from a probabilistic grammar similar to Ferrigno's bracket language, which defines sequences with multiple center-embedded dependencies. The model was trained at a fixed nesting depth, then tested on unseen sequences with other depths. Results showed that all models generalized well to unseen sequences with a smaller or equal depth, but failed to generalize to a greater nesting depth. Thus, once again, those networks did not recurse. In sum, insights from RNNs suggest that: (1) while behavior seems to support recursive processing, the underlying neural mechanism often tells a different story. (2) testing for genuine recursion requires generalization to deeper center-embedded structures. Those observations limit the conclusions that can be drawn from the Ferrigno's purely behavioral results, especially since those are based on training and test sets that share the same nesting depth. The authors did test for generalization, but only at a fixed depth, using novel sets of brackets (e.g. $<>$ ). Given that the monkeys received no instruction about which of these symbols should serve as a left or right bracket, it seems that they could only succeed by visual analogy to previous symbols, thus falling short of demonstrating an understanding of recursion.

In fact, Ferrigno et al.'s results may also be explained by an even simpler model of sequence memory, the Botvinick and Watanabe model [Botvinick and Watanabe, 2007]. In this model, each sequence item is attached to a memory 'slot' by neurons jointly sensitive to the product of item identity and ordinal position. The latter is encoded by neurons tuned to approximate number [Nieder and Dehaene, 2009]. Thus, the two training sequences are encoded as $1\{2(3) 4\}$ and $1\{2[3] 4\}$. When confronted with a generalization display containing only the symbols ( ) [ ], participants would pick, for each ordinal position, the item that occupies the closest location in working memory:

- For position 1, the closest item is in position 2, hence monkeys pick either ( or [.

- For position 2: monkey pick the left-over item, namely [ if ( was picked first.

- For position 3; two items are again possible: ) or ].

- For position 4, a single choice remains.

This strategy predicts an equal production of the "embedded" sequence ( [ ] ) and the "crossed" sequence ( [ ) ], exactly what the monkeys initially did. With more training, the monkeys may have become sensitive to the systematic pairing of ( ) and [ ] in the training sequences, with transition probabilities equal to one. Thus, overtrained monkeys would systematically produce ( ) or [ ] in the middle of the sequence, leading to purely "embedded" responding - again exactly as observed. This model also explains why, in humans, Ferrigno et al. found that the production of embedded sequences correlates with measures of working memory. This correlation would be unexpected if their task probed language-like recursive structures, because working memory and language rely on distinct brain circuits [Fedorenko et al., 2011], but makes sense according to the working-memory slot model.

In conclusion, we would all welcome a task capable of selectively probing animals' understanding of recursion. Alas, Ferrigno et al.'s task falls short of this ideal.

\section{References}

Stephen Ferrigno, Samuel J Cheyette, Steven T Piantadosi, and Jessica F Cantlon. Recursive sequence generation in monkeys, children, us adults, and native amazonians. Science Advances, 6(26):eaaz1002, 2020.

Matthew Botvinick and Takamitsu Watanabe. From numerosity to ordinal rank: a gain-field model of serial order representation in cortical working memory. Journal of Neuroscience, 27(32):8636-8642, 2007.

Mirac Suzgun, Sebastian Gehrmann, Yonatan Belinkov, and Stuart M Shieber. Memory-augmented recurrent neural networks can learn generalized dyck languages. arXiv preprint arXiv:1911.03329, 2019.

Fred Karlsson. Constraints on multiple center-embedding of clauses. Journal of Linguistics, 43(2):365-392, 2007.

Tal Linzen, Emmanuel Dupoux, and Yoav Goldberg. Assessing the ability of LSTMs to learn syntax-sensitive dependencies. Transactions of the Association for Computational Linguistics, 4:521-535, 2016.

Yair Lakretz, Germán Kruszewski, Theo Desbordes, Dieuwke Hupkes, Stanislas Dehaene, and Marco Baroni. The emergence of number and syntax units in LSTM language models. In Proceedings of NAACL, pages 11-20, Minneapolis, MN, 2019. 
Yair Lakretz, Dieuwke Hupkes, Alessandra Vergallito, Marco Marelli, Marco Baroni, and Stanislas Dehaene. Mechanisms for handling nested dependencies in neural-network language models and humans. Cognition, page 104699, 2021a.

Yair Lakretz, Stanislas Dehaene, and Jean-Rémi King. What limits our capacity to process nested long-range dependencies in sentence comprehension? Entropy, 22(4):446, 2020.

Yair Lakretz, Théo Desbordes, Jean-Rémi King, Benoît Crabbé, Maxime Oquab, and Stanislas Dehaene. Can rnns learn recursive nested subject-verb agreements? arXiv preprint arXiv:2101.02258, $2021 \mathrm{~b}$.

Andreas Nieder and Stanislas Dehaene. Representation of number in the brain. Annual review of neuroscience, 32 : 185-208, 2009.

Evelina Fedorenko, Michael K Behr, and Nancy Kanwisher. Functional specificity for high-level linguistic processing in the human brain. Proceedings of the National Academy of Sciences, 108(39):16428-16433, 2011. 